\title{
G Research Square \\ Urinary Phenols and Parabens Metabolites with Cardiovascular Disease in United States Adult
}

\section{Ting Yin}

The First Affiliated Hospital of Nanjing Medical University, Jiangsu Province Hospital

\section{Xu Zhu}

The First Affiliated Hospital of Nanjing Medical University, Jiangsu Province Hospital

\section{lokfai Cheang}

The first Affiliated Hospital of Nanjing Medical University, Jiangsu Province Hospital

\section{Yufei Zhou}

The First Affiliated Hospital of Nanjing Medical University, Jiangsu Province Hospital

\section{Shengen Liao}

The First Affiliated Hospital of Nanjing Medical University, Jiangsu Province Hospital

\section{Xinyi Lu}

The First Affiliated Hospital of Nanjing Medical University, Jiangsu Province Hospital

\section{Yanli Zhou}

The First Affiliated Hospital of Nanjing Medical University, Jiangsu Province Hospital

\section{Wenming Yao}

The First Affiliated Hospital of Nanjing Medical University, Jiangsu Province Hospital

\section{Xinli Li ( $\nabla$ xinli3267@njmu.edu.cn )}

The First Affiliated Hospital of Nanjing Medical Univeristy, Jiangsu Province Hospital https://orcid.org/0000-0002-7889-6359

\section{Haifeng Zhang}

The Affiliated Hospital of Nanjing Medical University, Jiangsu Province Hospital; Affiliated Suzhou Hospital of Nanjing Medical University, Suzhou Municipal Hospital

\section{Research Article}

Keywords: Phenols, Parabens, Cardiovascular disease, Weight quantile sum, NHANES

Posted Date: June 18th, 2021

DOI: https://doi.org/10.21203/rs.3.rs-562561/v1

License: (9) (1) This work is licensed under a Creative Commons Attribution 4.0 International License. Read Full License 
Version of Record: A version of this preprint was published at Environmental Science and Pollution Research on August 3rd, 2021. See the published version at https://doi.org/10.1007/s11356-021-155895 . 


\section{Abstract}

Environmental health has begun to examine the effects of higher-order chemical combinations. The current literature lacks studies exploring associations between multiple organic chemicals mixture and cardiometabolic disease (CVD). This study aimed to evaluate associations between urinary phenol and paraben metabolites and total and individual CVD among a nationally representative sample of adults in the US. This cross-sectional study analyzed 7 urinary chemicals detected among the general population from the 2005-2016 National Health and Nutrition Examination Survey (NHANES, $n=10,428$ ).

Multivariate logistic regression and weighted quantile sum (WQS) regression were applied to examine relationships between phenol and paraben metabolites, alone and combined, and total and individual CVD prevalence. WQS regression showed that phenol and paraben indices were independently correlated with total CVD (adjusted odds ratios [OR]: 1.16; 95\% confidence interval [Cl]:1.06-1.28; $P=0.002$ ), angina (adjusted OR: 1.30; 95\% Cl: 1.07-1.59; P = 0.009), and heart attack (adjusted OR: 1.30; 95\% Cl: 1.12-1.51, $\mathrm{P}<0.001)$. Urinary bisphenol A (URBPA, weight $=0.636$ ) was the most heavily weighted component in the total CVD model. Compared with the lowest quartile, URBPA (OR: 1.52; 95\% Cl: $1.20-1.91 ; \mathrm{P}=0.001$ ) levels in the highest quartile were independently associated with increased total CVD. Restricted cubic spline regression demonstrated positive correlations and non-linear associations between URBPA and both total CVD ( $P$ for nonlinearity $=0.032$ ) and individual CVD (heart attack; $P$ for nonlinearity $=0.031$ ). Our findings suggested that high combined levels of phenols and parabens are associated with an increased CVD risk, with the URBPA contributing the highest risk.

\section{Introduction}

Cardiovascular disease (CVD) is the leading cause of death and disability worldwide and is associated with substantial burdens on medical and health resources (GBD 2016 Causes of Death Collaborators, 2017; GBD 2016 DALYs and HALE Collaborators, 2017). The American Heart Association has predicted that approximately $43.9 \%$ of the US population will have CVD by 2030 (Benjamin et al., 2017). Therefore, exploring the modifiable environmental risk factors that contribute to CVD might provide insights into potential preventive measures. The environment is awash with synthetic chemicals from a variety of sources. Estimates suggest that $>30,000$ synthetic chemicals are in current use, among which at least 5,500 are produced at quantities $>100$ tons per year (Hartung, 2009). Persistent chemical use is common in food processing, packaging, chemical necessities, and the pharmaceutical industry, and the most commonly used chemicals include phenols and parabens. To date, numerous epidemiological and clinical studies have described the adverse effects of ubiquitous exposure to multiple phenols and parabens due to widespread use in food processing and packaging (Calafat et al., 2015). As a consequence, these chemicals have contaminated the surrounding environment and can be transformed into corresponding metabolites, which can result in high concentrations in animal and human tissues and bodily fluids (Wilhelm et al., 2003; llaria et al., 2020). CVD has been associated with higher concentrations of phenols and parabens among individuals with lower socioeconomic status (Ruiz et al., 2018), lower 
body mass index (BMI) (Liu et al., 2017), and exposure to cigarette smoke, although not all studies have reported consistent results (Quiros-Alcala et al., 2018; Julia et al., 2020).

Among phenolic chemicals, bisphenol A (BPA) is the chemical produced at the highest volume and is used extensively and globally in the production of polycarbonate plastics, which has resulted in regulatory bans in an attempt to reduce BPA use and promote the increased use of replacement compounds (Ilaria et al.,2020). Benzophenone-3 (BP3) is a chemical ultraviolet filter that is commonly used to absorb ultraviolet rays. Non-occupational exposures to dichlorophenol (DCP) occur through the inhalation of contaminated air or the ingestion of contaminated food or chlorinated water (Zamoiski et al., 2015). 2,4-DCP and 2,5-DCP are byproducts produced by wastewater treatment, waste incineration, and wood pulp bleaching and are produced as metabolites of some organochlorine pesticides (Dodson et al., 2012). Triclosan (TCS) is an antimicrobial chemical that is widely used in products such as antibacterial soaps, toothpaste, pens, diaper bags, and medical devices, which has been associated with the development of bacterial resistance via target site modification in a dose-dependent manner (Nietch et al., 2013). Some animal-based studies have revealed that the byproducts of phenol decomposition can affect mitochondrial function (Shim et al., 2016), disrupt calcium signaling (Cherednichenko et al., 2012), damage reproductive and thyroid hormone production (Stephanie et al., 2018), alter immunological parameters (Yueh, 2014) and potentially reduce cardiovascular function (Cherednichenko et al., 2012; Ahn et al., 2008). Meanwhile, parabens have a lipophilic character and are widely used as additives in germicides and as anti-corrosive agents, mainly including butyl-p-hydroxybenzoate (BUP), ethyl 4hydroxybenzoate (EPB), methyl 4-hydroxybenzoate (MPB), and propyl 4-hydroxybenzoate (PPB) (Ferguson et al., 2016). The lipophilicity of these exogenous chemicals allows for toxic hydrophilic species to permeate lipophilic membranes, inducing CVD (Harold and Zeliger, 2013). Additionally, the accumulation of lipophilic phenols and parabens due to prolonged exposure and the total serum load of lipophilic species might be a determining factor that triggers CVD (Harold and Zeliger, 2013). Singlechemical pollutant analyses examining individual phenols and parabens have indicated that these singular chemicals are associated with increased risks of obesity (Traci et al., 2020; Lesliam et al., 2018; Thomas et al.,2020), type 2 diabetes (Andrea et al., 2019; Robert et al., 2019), hypertension (Andreia et al., 2019), and cardio-related risk factors (Symielle et al., 2020).

Although studies have shown various correlations between exposure to individual metabolites as separate chemicals (Chris et al., 2020), each has been insufficient to explain the real-world pathological outcomes. The field of environmental health has increasingly moved toward studying the effects of higher-order chemical combinations (Neter et al., 1996). Recent evidence has documented an increase in the occurrence of acute exacerbations in cardiovascular events and an increase in CVD chronic morbidity following exposure to multiple synthetic chemicals (Bhatnagar, 2006; Calafat et al., 2010). Weighted quantile sum (WQS) has been recommended as a multiple regression method that can be used to analyze the synergistic effects of multiple chemical exposures on CVD outcomes to identify the effects of mixed exposures and identify the most influential chemicals within the mixture (Carrico et al., 2015). 
The aim of the present study was to explore the effects of multiple exposures to phenols and parabens on CVD outcomes by analyzing the demographic characteristics and corresponding urinary chemical marker data collected in the National Health and Nutrition Examination Survey (NHANES) from 2005 to 2016.

\section{Material And Methods}

\section{Study population and design}

The NHANES is administered by the National Center for Health Statistics (NCHS) at the US Centers for Disease Control and Prevention (CDC). NHANES uses a multi-stage, stratified, probability sampling design to obtain unbiased national health information (detailed sample design: https://wwwn.cdc.gov/nchs/nhanes/tutorials/module2.aspx). Data from NHANES surveys for 20052016 includes exposures to multiple synthetic chemicals among the noninstitutionalized US population (NCHS) (Fangjian and Timothy, 2016). None of the authors of the current study were involved in the collection of data or the production of the database in use.

A total of 60,936 subjects were enrolled in the initial analysis. Participants missing data for phenols and parabens $(n=45,209)$, those pariticipants younger than 20 years and missing data regarding CVD outcomes $(n=5,299)$ were excluded from the present analysis. In total, 10,428 subjects with complete data on exposure to multiple phenols and parabens, primary covariates, and CVD outcomes were included for further analysis (Fig. S1).

\section{Measurement of chemical exposures}

Urinary samples were collected and stored at $-20^{\circ} \mathrm{C}$ until they were sent to the National Center for Environmental Health, where they were analyzed by the Organic Analytical Toxicology Branch. A total of 9 chemicals, BPA, BP3, 2,4-DCP, 2,5-DCP, TCS, BUP, EPB, MPB, and PPB were measured. The limits of detection (LOD) for BPA, BP3, 2,4-DCP, 2,5-DCP, TCS, MPB, and PPB were $0.2 \mathrm{ng} / \mathrm{mL}, 0.4 \mathrm{ng} / \mathrm{mL}, 1.0$ $\mathrm{ng} / \mathrm{mL}, 0.1 \mathrm{ng} / \mathrm{mL}, 1.7 \mathrm{ng} / \mathrm{mL}, 0.1 \mathrm{ng} / \mathrm{mL}$, and $0.1 \mathrm{ng} / \mathrm{mL}$, respectively (Supplement Table 1 ). The concentrations of BUP and EPB were not incorporated in further analyses due to low detectable concentrations.

The concentrations and distributions of the 7 remaining urinary chemical metabolites were analyzed. Urinary BPA, BP3, 2,4-DCP, and 2,5-DCP metabolites were extracted using online solid-phase extraction (SPE) and measured by high-performance liquid chromatography and tandem mass spectrometry (MS/MS). Urinary TCS, MPB, and PPB metabolites were extracted using online SPE and measured by high-performance liquid chromatography-isotope dilution MS/MS (34). All concentrations below the LOD were replaced by the LOD value divided by the square root of $2(\mathrm{LOD} / \sqrt{2})$. The detailed protocol is described elsewhere (NHANES 2011-2012: Environmental Phenols \& Parabens Data Documentation, Codebook, and Frequencies (cdc.gov)). All urinary measurements were adjusted for creatinine to account for differences in kidney function. 


\section{Assessment of CVD outcomes}

Participants responded to questions related to medical conditions and CVD outcomes prior to undergoing a physical examination, which was documented by a Computer-Assisted Personal Interviewing (CAPI) system. Responses to follow-up questions related to CVD-specific medications and healthcare were also ascertained. Self-reported diagnosed and borderline diabetes/high blood pressure assessments were combined as 'diabetes/hypertension' for the purposes of this analysis.

Participants aged $\geq 20$ years were asked, "Has a doctor or other health professional ever told you that you have..." congestive heart failure, coronary heart disease, angina, heart attack, or stroke. "Cardiovascular disease" (CVD) was defined as any reported diagnosis of congestive heart failure (CHF), coronary heart disease (CHD), angina, heart attack, or stroke. The data for each CVD outcome were collected for further analyses of associations with the 7 assessed urinary chemical metabolites.

\section{Covariates}

Potential confounders of the CVD outcome measure, including demographic characteristics (age, sex, educational levels, race/ethnicity, and poverty income ratio [PIR]), lifestyle characteristics (BMI, smoking, and alcohol use), comorbidities (diabetes mellitus and hypertension), and urine creatinine (Ucrea) levels were collected. BMI was calculated as the ratio of weight in kilograms to height in meters squared $\left(\mathrm{kg} / \mathrm{m}^{2}\right)$. Ucrea data were collected by interview or laboratory detection performed by NHANES.

Covariates details were as follows: (1) age was modeled continuously; (2) sex was dichotomized as male or female; (3) education level was coded as blow/above high school and high school (or K-12 aged (6-19 years old), $\leq$ high school/GED, some college, $\geq$ Bachelor Degree); (4) the family PIR was assessed as the ratio of family income to the poverty threshold, poverty is characterized as a family PIR below 1 (Lesliam et al., 2018); (5) a smoker was identified as someone who smoked at least 100 cigarettes in his lifetime, alcohol use were categorized as never drinker, former drinker, current drinker; (6) diabetes mellitus (DM) and hypertension were assessed and determined by the questionnaire administered by NHANES personnel (Lesliam et al., 2018); Ucrea (mg/dL) concentrations were measured from the urine samples using an automated colorimetric method based on a modified Jaffe reaction on a Beckman Synchron AS/ASTRA clinical analyzer (Beckman Instruments, Inc., Brea, CA) and were used to correct for urine dilution in our statistical models.

\section{Statistical analyses}

A total of 7 urinary phenol and paraben metabolites (BPA, BP3, 2,4-DCP, 2,5-DCP, TCS, MPB, and PPB) were analyzed for their contributions to the risk of categorized CVD outcomes (CHD, CHF, heart attack, stroke, and angina).

The urinary chemical metabolite concentrations were log-transformed to normalize their distributions for further analysis. The Kolmogorov-Smirnov test was used to assess the normality of continuous 
variables. Continuous variables are expressed as the mean (standard deviation [SD]) and were compared using unpaired t-tests. Categorical or dichotomous variables are expressed as absolute values (percentages) and were compared using $\chi^{2}$ statistics. Pearson's correlation coefficients were calculated among all urinary phthalate exposures. Multivariable logistic regression models were used to calculate odds ratios (ORs) and 95\% confidence intervals (Cls) to assess the prevalence of total and individual CVDs associated with urinary phenols, parabens, and TCS metabolites. The metabolite levels of urinary phenols, parabens, and TCS were divided into quartiles, and the lowest quartile was used as the reference category.

Regression models were adjusted for potential confounders, including age, sex, race, education levels, and poverty, which a previous study (Traci et al., 2020) reported being associated with urinary metabolite concentrations, and Ucrea was used to adjust for urine concentration (Ferguson et al., 2016).

Initial adjustment was for the following: age; sex; education; Advanced models were additionally adjusted for smoking, drinking, BMI, diabetes and hypertension history, and urinary creatinine concentration; Fully adjusted models were adjusted as advanced adjusted models plus other urinary 6 chemicals. Association between urinary chemicals metabolites concentration and CVDs were contacted. The calculate odds ratios (ORs) and 95\% confidence intervals (Cls) used in multivariable logistic regression.

WQS regression was used to assess associations between mixtures of chemical exposures and CVD. Each exposure variable is assigned a weight within the index, which indicates the importance of the variable within the mixture (Bhatnagar, 2006; Han et al., 2016). WQS models were run using the gWQS v.2.0.0 in R v.3.6.1. Within the gWQS function, the reported results were estimated by specifying deciles for exposure weighting, with $40 \%$ of the data used as the test set and the remaining $60 \%$ used as the validation set, 1,000 bootstrap repetitions, the random seed set to 2016 , and a binomial distribution used for the general linear model. In each case, the weights were estimated by pooling only the effects in the positive or negative direction as constrained. Individual chemical weights $\geq 0.1$ were considered the most important. The value of 0.1 was chosen for ease of comparison across models, including different numbers of chemicals. Restricted cubic splines with knots were used at the 5th, 10th, 50th, and 90th percentiles for multiple phenols and parabens distributions with the highest weight proportions from the above results to further explore the dose-response curves between multiple phenols, parabens, and total CVD. Significance was set at $P<0.05$ (two-sided).

\section{Results}

\section{Study population characteristics according to total CVD status}

The final analysis included 10,428 adults (male, $n=5,084$; female, $n=5,344$, mean age 49 years). The demographic characteristics of participants included in the final analysis are presented in Table 1. Based on the occurrence of CVD, the population was divided into a CVD group ( $N=1,119,10.7 \%)$ and a non-CVD group ( $N=9,309,89.3 \%)$. The CVD group was older, with a higher proportion of men, a lower average 
education level, a less Hispanic composition, a higher poverty level, a higher average BMI, a higher incidence of smokers, and a higher incidence of both DM and hypertension, which were significantly different from the non-CVD group (all $\mathrm{P}<0.05$ ).

\section{Phenols and parabens metabolites and correlation analysis}

The distributions and limits of the 7 detected chemical metabolites are shown in Table S1. The detection rate for URTCS was $74.5 \%$, and the detection rates for all other chemical metabolites were above $89 \%$. Correlation analyses of the urinary chemical metabolites showed that some of the metabolites were moderately correlated with the other 6 metabolites (Pearson's $r \geq 0.3$ ). Strong positive correlations were found between URMPB and URPPB (correlation coefficient, $r=0.82, P<0.001$ ) and between UR2,4-DCP, and UR2,5-DCP $(r=0.83, P<0.001)$. A moderate positive correlation was identified between Ucrea and URBPA $(r=0.56, P<0.001)$ (Fig. 1).

\section{Associations between the urinary phenols and parabens metabolites and total CVD}

Our findings showed that in Model 1, URBP3, URBPA, and URTCS were associated with an increased prevalence of total CVD. The 7 urinary metabolites were further included as covariates to prevent false positives induced by multiple corrections in Models 2 and 3 . The results showed that compared with the levels in the lowest quartile, the URBPA (OR: 1.52; $95 \% \mathrm{Cl}$ : $1.20-1.91, \mathrm{P}$ for trend $=0.001$ ) levels in the highest quartile were independently associated with an increased prevalence of total CVD. No significant correlations were identified between the other 6 urinary metabolites and total CVD (Table 2).

\section{WQS regression analysis of urinary phenols and parabens metabolites mixtures and both total/individual CVD}

The adverse relationships between urinary phenol and paraben mixtures and the prevalence rates of total and individual CVD were analyzed using WQS regression analysis. The 7 chemical metabolite index was independently correlated with total CVD (adjusted OR: 1.16; 95\% Cl: 1.06-1.28, P = 0.002), angina (adjusted OR: 1.30; 95\% Cl: 1.07-1.59, P = 0.009), and heart attack (adjusted OR: 1.30; $95 \% \mathrm{Cl}: 1.12-1.51, \mathrm{P}<0.001)$ but was not correlated with the prevalence rates of $\mathrm{CHF}, \mathrm{CHD}$, or stroke (Table 3).

URBPA (weight $=0.636$ ) was the most heavily weighted component in the total CVD model. UR2,5-DCP (weight $=0.320$ ) was the only other component with a weight $>0.1$. We also calculated the weight of each environmental chemical for the individual CVD model (Fig. 2). The URBPA weights were $0.347,0.262$, $0.376,0.615$, and 0.588 for the CHF, CHD, angina, heart attack, and stroke models, respectively. UR2,5-DCP was also heavily weighted, with values of $0.116,0.328,0.453,0.204$, and 0.588 for the CHF, CHD, angina, heart attack, and stroke models, respectively (Table S2).

\section{Associations between URBPA and the prevalence rates of individual and total CVD}


The relationship between URBPA and individual CVD was further analyzed using multiple logistic regression, as shown in Table 3. After adjusting for multivariate models, URBPA levels in the highest quartile remained significantly associated with increased risks of heart attack (OR: $1.54 ; 95 \% \mathrm{Cl}: 1.18-$ 2.02, $\mathrm{P}$ for trend $=0.003$ ) and stroke (OR: $1.52 ; 95 \% \mathrm{Cl}: 1.12-2.07$, P for trend $=0.029)$. In a restricted cubic spline with a multivariate logistic regression model, the URBPA level demonstrated non-linear associations and positive correlations with total CVD ( $P$ for nonlinearity $=0.032$; Fig. 3 ) and heart attack ( $P$ for nonlinearity $=0.031$; Fig. 4). In addition, URBPA levels had linear associations and positive correlations with other individual CVDs (angina, $\mathrm{P}$ for nonlinearity $=0.326$; stroke, $\mathrm{P}$ for nonlinearity $=$ 0.870; and CHF, P for nonlinearity = 0.689; Fig. S2).

\section{Discussion}

Data were collected from 10,428 adults as part of a prospective, nationally representative US cohort between 2005 and 2016, and logistic regression and WQS regression were used to investigate the associations between urinary phenol and paraben compounds and the prevalence rates of total and individual CVD. The multipollutant model results were relatively consistent but non-interchangeable. The present study found that the 7 chemical metabolite index was independently correlated with total CVD, angina, and heart attack. URBPA was the most heavily weighted chemical in the total CVD model, and the concentration of URBPA was positively and non-linear associated with CVD risk. In addition, URBPA concentration also displayed linear associations and positive correlations with individual CVDs, including CHF, angina, and stroke.

We investigated available phenol and paraben chemicals, including BPA, BP3, 2,4-DCP 2,5-DCP, TCS, MPB, and PPB, which are commonly used for daily necessities, industry, and agricultural purposes. In our study, WQS regression analyses were performed at the total population level, and systematic analysis was applied to the 7 metabolic compounds, which allowed correlations between the phenol and paraben mixtures and the prevalence rates for total and individual CVDs to be identified that could not be observed using conventional approaches. Combinations of chemical exposures have effects on thyroid signaling, metabolism, oxidative stress, and obesity and affect the regulation of cardiovascular function, which has been demonstrated by both animal and human studies (Traci et al., 2020; Ferguson et al., 2016). However, studies have rarely analyzed the effects of both phenols and parabens on CVD in the general population using higher-order combinations of chemicals. Our study identified an association between the exposure index and CVD outcomes through a lower dimensional plot, linking these biomarker measurements to exposure and internal concentrations, which provides a better understanding of the metabolism of phenol and paraben mixtures in humans.

In the study seven chemical mixture index associated with CVD, not each chemicals. Chemicals at low concentrations that individually have no observable effects can act together to produce a joint effect, causing a wide range of outcomes exist in response to the presence of various organic chemicals in the water, air, and soil (Benjamin et al., 2017; Wilhelm et al., 2003). Our study used WQS regression to evaluate correlations with a chemical mixture index comprising 7 correlated, high-dimensional 
metabolites. When analyzing interactions between chemical exposures and mixed predictors, the WQS model has high sensitivity and specificity, providing deeper insights into correlations between the phenol and paraben index calculations and simultaneously quantifying the relative importance of each urinary metabolite. Strong positive correlations in the study were found between MPB and PPB and between 2,4DCP and 2,5-DCP. Others exhibited a moderate correlation. Each chemical exerts specific effects on health through interactions with various cellular components, and similar chemical properties determine the internal metabolic response (Nietch et al., 2013; Symielle et al., 2020; Bhatnagar, 2006). The most heavily weight chemical has the most influential impact on the accumulated chemical mixture index. The WQS index identifies adverse chemicals based on non-negligible weights (Chris et al., 2020). BPA was the heaviest weighted chemical associated with total CVD in our study, within the total 7-metabolite mixture.

In addition to BPA, TCS is another chemical that was identified as a significant factor in the multivariable logistic regression after adjusting for common cardiovascular risk factors. The other 5 chemicals were negatively associated with total and individual CVD. In 2017, BPA was included in the European Chemical Agency (ECHA) Candidate List of substances of very high concern and was identified as being highly associated with CVD (GBD 2016 Causes of Death Collaborators, 2017; Liu et al., 2017). BPA is an estrogenic chemical that promotes vasodilation by stimulating prostacyclin and nitric oxide synthesis and decreasing the production of vasoconstrictor agents (Han, et al., 2016). Other potential physiological effects with implications for cardiometabolic health include improvements in endothelial function, platelet function, and glucose and lipid metabolism (Habauzit et al., 2012; Cicero et al., 2017). The underlying mechanisms of action are thought to be related to the ability of phenols to modulate insulin resistance, activate $\mathrm{K}+$ channels, block the activation of Ca2 + channels (Kerimi et al., 2016; Krga et al., 2016), and activate CD4 ${ }^{+} T$ cells and CD36 to aggravate inflammatory factor expression, which can lead to cardiac fibrosis and atherosclerosis (Katelyn et al., 2019; Stéphanie et al., 2019). Through these mechanisms, BPA participates in cardiac hypoperfusion and vascular-related dysfunction (Reventun et al., 2020). TCS and its 2 derivatives (2,4-DCP and 2,5- DCP) are priority pollutants that coexist in the aquatic system and have been reported to induce cardiac toxicity in vivo (Danting et al., 2020). Our investigations in the general population provide additional epidemiological evidence to indicate the adverse effects of these 7 chemicals and contribute to the foundation for advanced studies of environmentally hazardous substances.

Our study has certain limitations. First, the use of a single urine sample may not efficiently reflect the average level of weekly or monthly exposure to chemical compounds; however, a single urinary analysis provided reliable exposure measures for BPA from a mixture of chemicals, likely due to regular exposure in food and daily consumables. Second, WQS regression was required to separate positive and negative models, which restricted its ability to evaluate the overall impact of all metabolites (Carrico et al., 2015). However, WQS regression has the ability to address high-dimensional mixtures and inevitably collinear data to explore environmental toxicants that represent public health issues. Finally, the possible causation in the relationship between urinary phenol and paraben metabolites and mixtures and CVD must be further validated in prospective cohort studies. 


\section{Conclusions}

Our findings suggested that urinary phenol and paraben metabolite mixtures were significantly and positively associated with CVD. The effects of multiple exposures to urinary chemicals on CVD differ in magnitude across the chemicals analyzed, with the greatest influence contributed by URBPA. Additional methods are required when mixture analysis is used because these results show the significance of cooccurring exposures.

\section{Declarations}

\section{Ethical Approval and consent to Participate}

The consent form was signed by participants in the survey, and participants consented to storing specimens of their blood for future research. The CDC/NCHS Ethics Review Board approved the NHANES study and gave approval for public dissemination.

\section{Consent to Publish}

Not applicable. There is no individual level data in our publication.

\section{Authors contributions}

Ting Yin and Xu Zhu designed and conceived the study proposal, conducted statistical analyses, and draft of the manusript. Ting Yin, lokfai Cheang and Shengen Liao critically revised the manuscript. Yufei Zhou, Xinyi Lu and Yanli Zhou performed the bibliographic research, analyzed the data. Haifeng Zhang and Wenming Yao conceived and led the design of the study and model. Xinli Li revised the manuscript. All authors have read and approved the final article.

\section{Funding}

Not applicable. No funding was received to assist with the preparation of this manuscript.

\section{Competing Interests}

The authors declare that they have no known competing financial interests or personal relationships that could have appeared to influence the work reported in this paper.

\section{Ackonwlegements}

The authors would like to thank the CDC for conducting NHANES survey and NCHS staff for providing us with consultation on the application of survey weights. This research did not receive any specific grant from funding agencies in the public, commercial, or not-for-profit sectors.

\section{Availability of data and materials}


The datasets used and analyzed during the current study are available from https://wwwn.cdc.gov/nchs/nhanes/tutorials/module2.aspx.

\section{References}

1. Ahn KC, Zhao B, Chen J, Cherednichenko G, Sanmarti E, Denison MS, et al., 2008. In vitro biologic activities of the antimicrobials triclocarban, its analogs, and triclosan in bioassay screens: Receptorbased bioassay screens. Environ Health Persp 116:1203-1210.

2. Andrea Bellavia, Yu-Han Chiu, Florence M. Brown, Lidia Mínguez-Alarcón, Jennifer B. Ford, John Petrozza, et al., 2019. Urinary concentrations of parabens mixture and pregnancy glucose levels among women from a fertility clinic. Environ Res. Jan; 168: 389-396.

3. Andreia Gomes, Carole Oudot, Alba Macià, Alexandre Foito, Diogo Carregosa, Derek Stewart, et al., 2019. Berry-Enriched Diet in Salt-Sensitive Hypertensive Rats: Metabolic Fate of (Poly)Phenols and the Role of Gut Microbiota. Nutrients. Nov; 11(11): 2634.

4. Benjamin, E.J., Michael J. Blaha, Stephanie E. Chiuve, Mary Cushman, Sandeep R. Das, Rajat Deo, et al., 2017. Heart disease and stroke statistics-2017 update: a report from the American Heart Association. Circulation 135, e146-e603.

5. Bhatnagar A., 2006. Environmental cardiology: studying mechanistic links between pollution and heart disease. Circ Res. Sep 29; 99(7):692-705.

6. Calafat AM, Valentin-Blasini L, Ye X., 2015. Trends in Exposure to Chemicals in Personal Care and Consumer Products. Current environmental health reports. ;2(4):348-55.

7. Cherednichenko G, Zhang R, Bannister RA, Timofeyev V, Li N, Fritsch EB, et al., 2012. Triclosan impairs excitation-contraction coupling and $\mathrm{Ca} 2+$ dynamics in striated muscle. Proc Natl Acad Sci U S A 109: 14158-14163.

8. Chris Gennings, Paul Curtin, Ghalib Bello, Robert Wright, Manish Arora, and Christine Austina, 2020. Lagged WQS regression for mixtures with many components Environ Res. Jul; 186: 109529.

9. Calafat AM, Ye X, Wong LY, Bishop AM, Needham LL., 2010. Urinary concentrations of four parabens in the U.S. population: NHANES 2005-2006. Environ Health Perspect; 118: 679-685.

10. Carrico, C., Gennings, C., Wheeler, D.C., Factor-Litvak, P., 2015. Characterization of weighted quantile sum regression for highly correlated data in a risk analysis setting. J. Agric. Biol. Environ. Stat. 20, $100-120$.

11. Cicero AF, Fogacci F, Colletti A., 2017. Food and plant bioactives for reducing cardiometabolic disease risk: an evidence based approach. Food Funct.;(6):2076-2088.

12. Danting Wang, Yuhuan Zhang, Jieyi Li, Randy A Dahlgren, Xuedong Wang, Haishan Huang, et al., 2020. Risk assessment of cardiotoxicity to zebrafish (Danio rerio) by environmental exposure to triclosan and its derivatives. Environ Pollut. Oct;265 (Pt A):114995.

13. Dodson RE, Nishioka M, Standley LJ, Perovich LJ, Brody JG, Rudel RA., 2012. Endocrine disruptors and asthma-associated chemicals in consumer products. Environmental health perspectives; 
120:935-943.

14. Fangjian Guo, W. Timothy Garvey, 2016. Trends in Cardiovascular Health Metrics in Obese Adults: National Health and Nutrition Examination Survey (NHANES), 1988-2014 J Am Heart Assoc. Jul; 5(7): e003619.

15. Ferguson KK, Cantonwine DE, McElrath TF, Mukherjee B, Meeker JD. 2016. Repeated measures analysis of associations between urinary bisphenol-A concentrations and biomarkers of inflammation and oxidative stress in pregnancy. Reprod Toxicol. 2016 Dec; 66():93-98.

16. GBD 2016 Causes of Death Collaborators, 2017. Global, regional, and national age-sex specific mortality for 264 causes of death, 1980-2016: a systematic analysis for the Global Burden of Disease Study 2016. Lancet; 390:1151-1210.

17. GBD 2016 DALYs and HALE Collaborators, 2017. Global, regional, and national disability-adjusted life-years (DALYs) for 333 diseases and injuries and healthy life expectancy (HALE) for 195 countries and territories, 1990-2016: a systematic analysis for the Global Burden of Disease Study 2016. Lancet; 390:1260-1344.

18. Hartung T, 2009. Toxicology for the twenty-first century. Nature. Jul 9; 460(7252):208 - 12.

19. Han C, Hong YC. Bisphenol A, 2016. Hypertension, and Cardiovascular Diseases: Epidemiological, Laboratory, and Clinical Trial Evidence. Curr Hypertens Rep. Feb; 18(2):11.

20. Habauzit V, Morand C., 2012. Evidence for a protective effect of polyphenols containing foods on cardiovascular health: an update for clinicians. Ther Adv Chronic Dis.;3:87-106.

21. Harold I. Zeliger. 2013 Lipophilic chemical exposure as a cause of cardiovascular disease. Interdiscip Toxicol. Jun; 6(2): 55-62.

22. Ilaria Cimmino, Francesca Fiory, Giuseppe Perruolo, Claudia Miele, Francesco Beguinot, Pietro Formisano, et al., 2020. Potential Mechanisms of Bisphenol A (BPA) Contributing to Human Disease. Int J Mol Sci. Aug; 21(16): 5761.

23. Julia B. Ward, Sarah S. Casagrande, Catherine C. Cowie., 2020. Urinary phenols and parabens and diabetes among US adults, NHANES 2005-2014. Nutr Metab Cardiovasc Dis. May 7;30(5):768-776.

24. Kerimi A, Williamson G., 2016. At the interface of antioxidant signalling and cellular function: key polyphenol effects. Mol Nutr Food Res. ;60(8):1770-1788.

25. Krga I, Milenkovic D, Morand C, Monfoulet LE., 2016. An update on the role of nutrigenomic modulations in mediating the cardiovascular protective effect of fruit polyphenols. Food Funct.;7(9):3656-3676.

26. Katelyn Ann Bruno, Jessica Elizabeth Mathews, Alex Lingyun Yang, J. Augusto Frisancho, Ashley Jennie Scott, Henry David Greyner, et al., 2019. BPA Alters Estrogen Receptor Expression in the Heart After Viral Infection Activating Cardiac Mast Cells and T Cells Leading to Perimyocarditis and Fibrosis. Front Endocrinol (Lausanne).; 10: 598.

27. Liu B, Lehmler HJ, Sun Y, Xu G, Liu Y, Zong G, et al., 2017. Bisphenol A substitutes and obesity in US adults: analysis of a population-based, cross-sectional study. Lancet Planet Health. Jun; 1(3): e114e122. 
28. Lesliam Quirós-Alcalá, Jessie P. Buckley, and Meleah Boyle, 2018. Parabens and measures of adiposity among adults and children from the U.S. general population: NHANES 2007-2014. Int J Hyg Environ Health. May; 221(4): 652-660.

29. Neter J, Kutner M, Nachtsheim C, Wasserman W, 1996. Applied Linear Statistical Models, fourth ed. (Chicago, Irwin:)

30. Nietch CT, Quinlan EL, Lazorchak JM, Impellitteri CA, Raikow D, and Walters D. 2013. Effects of a chronic lower range of triclosan exposure on a stream mesocosm community. Environ Toxicol Chem 32: 2874-2887.

31. P. Reventun, S. Sanchez-Esteban, A. Cook, I. Cuadrado, C. Roza, R. Moreno-Gomez-Toledano, et al., 2020. Bisphenol A induces coronary endothelial cell necroptosis by activating RIP3/CamKII dependent pathway. Sci Rep. ; 10: 4190.

32. Quiros-Alcala L., Buckley J.P., Boyle M., 2018. Parabens and measures of adiposity among adults and children from the U.S. general population: NHANES 2007-2014. Int J Hyg Environ Health; 221: pp. 652-660.

33. Ruiz D, Becerra M, Jagai JS, Ard K, Sargis RM., 2018. Disparities in Environmental Exposures to Endocrine-Disrupting Chemicals and Diabetes Risk in Vulnerable Populations. Diabetes Care. Jan; 41(1):193-205.

34. Robert M. Sargis, and Rebecca A. Simmons, 2019. Environmental neglect: endocrine disruptors as underappreciated but potentially modifiable diabetes risk factors. Diabetologia. Oct; 62(10): 18111822.

35. Recommendations for blood pressure measurement in humans and experimental animals: Part 1: blood pressure measurement in humans: a statement for professionals from the Subcommittee of Professional and Public Education of the American Heart Association Council on High Blood Pressure Research.

36. Shim J, Weatherly LM, Luc RH, Dorman MT, Neilson A, Ng R, et al., 2016. Triclosan is a mitochondrial uncoupler in live zebrafish. J Appl Toxicol 36:1662-1667.

37. Stephanie K. Bopp, Robert Barouki, Werner Brack, Silvia Dalla Costa, Jean-Lou C.M. Dorne, Paula E. Drakvik, et al., 2018. Current EU research activities on combined exposure to multiple chemicals. Environ Int. Nov; 120: 544-562.

38. Symielle A. Gaston, Linda S. Birnbaum, and Chandra L. Jackson, 2020. Synthetic Chemicals and Cardiometabolic Health across the Life Course among Vulnerable Populations: A Review of the Literature from 2018 to 2019. Curr Environ Health Rep. Mar; 7(1): 30-47.

39. Stéphanie Chacar, Joelle Hajal, Youakim Saliba, Patrick Bois, Nicolas Louka, Richard G. Maroun, et al., 2019. Long-term intake of phenolic compounds attenuates age-related cardiac remodeling, Aging Cell. Apr; 18(2): e12894.

40. Thomas P. van der Meer, Martijn van Faassen, André P. van Beek, Harold Snieder, Ido P. Kema, Bruce H. R. Wolffenbuttel, et al., 2020. Exposure to Endocrine Disrupting Chemicals in the Dutch general population is associated with adiposity-related traits. Sci Rep. ; 10: 9311. 
41. Traci N. Bethea, Amelia K. Wesselink, Jennifer Weuve, Michael D. McClean, Russ Hauser, Paige L. Williams, et al., 2020. Correlates of exposure to phenols, parabens, and triclocarban in the Study of Environment, Lifestyle and Fibroids J Expo Sci Environ Epidemiol. 2020 Jan; 30(1): 117-136.

42. Wilhelm M, Ewers U, Schulz C., 2003. Revised and new reference values for some persistent organic pollutants (POPs) in blood for human biomonitoring in environmental medicine. Int $\mathrm{J}$ Hyg Environ Health. Jun; 206(3):223-9.

43. Yueh MF, Taniguchi K, Chen S, Evans RM, Hammock BD, Karin M, et al., 2014. The commonly used antimicrobial additive triclosan is a liver tumor promoter. Proc Natl Acad Sci. 111:17200-17205.

44. Ye X, Kuklenyik Z, Needham LL, Calafat AM., 2005. Automated on-line column-switching HPLCMS/MS method with peak focusing for the determination of nine environmental phenols in urine. Anal Chem. ;77(16):5407-13.

45. Zamoiski RD, Cahoon EK, Michal Freedman D, Linet MS., 2015. Self-reported sunscreen use and urinary benzophenone-3 concentrations in the United States: NHANES 2003-2006 and 2009-2012. Environ Res. Oct; 142():563-7.

\section{Tables}

Table 1. Characteristics of the study populations according to total CVD status. 


\begin{tabular}{|lllll|}
\hline Variable & Total $(\mathrm{n}=10428 \rrbracket$ & Non-CVD $(\mathrm{n}=9309)$ & CVD $(\mathrm{n}=1119)$ & Pvalue \\
\hline Age, years & $49.0(17.8)$ & $47.0(17.3)$ & $65.7(13.1)$ & $<0.001$ \\
\hline Male, \% & $5084(48.8 \%)$ & $4443(47.7 \%)$ & $641(57.3 \%)$ & $<0.001$ \\
\hline Education level, \% & & & & $<0.001$ \\
\hline Below high school & $2698(25.9 \%)$ & $2300(24.7 \%)$ & $398(35.6 \%)$ & \\
\hline High school & $2406(23.1 \%)$ & $2142(23.0 \%)$ & $264(23.6 \%)$ & \\
\hline Above high school & $5324(51.1 \%)$ & $4867(52.3 \%)$ & $457(40.8 \%)$ & \\
\hline Race/ethnicity, \% & & & & $<0.001$ \\
\hline Mexican American & $1636(15.7 \%)$ & $1512(16.2 \%)$ & $124(11.1 \%)$ & \\
\hline Other Hispanic & $995(9.5 \%)$ & $916(9.8 \%)$ & $79(7.1 \%)$ & \\
\hline Non-Hispanic White & $4438(42.6 \%)$ & $3846(41.3 \%)$ & $592(52.9 \%)$ & \\
\hline Non-Hispanic Black & $2285(21.9 \%)$ & $2030(21.8 \%)$ & $255(22.8 \%)$ & \\
\hline Other race & $1074(10.3 \%)$ & $1005(10.8 \%)$ & $69(6.2 \%)$ & $<0.001$ \\
\hline Poverty, \% & $2290(22.0 \%)$ & $2009(21.6 \%)$ & $281(25.1 \%)$ & 0.007 \\
\hline Body mass index, kg/m2 & $29.1(6.9)$ & $28.9(6.8)$ & $30.5(7.0)$ & $<0.001$ \\
\hline Ucrea, mg/dL & $111.0[65.0-169.0]$ & $112.0[65.0-171.0]$ & $105.0[62.0-155]$ & 0.001 \\
\hline Smoker, \% & $4663(44.7 \%)$ & $3980(42.8 \%)$ & $683(61.0 \%)$ & $<0.001$ \\
\hline Alcohol user, \% & $7427(71.2 \%)$ & $6706(72.0 \%)$ & $721(64.4 \%)$ & $<0.001$ \\
\hline Diabetes mellitus, \% & $1290(12.4 \%)$ & $918(9.9 \%)$ & $372(33.2 \%)$ & $<0.001$ \\
\hline Hypertension, \% & $3715(35.6 \%)$ & $2887(31.0 \%)$ & $828(74.0 \%)$ & $<0.001$ \\
\hline
\end{tabular}

Data are presented as mean (SD) or median [interquartile range], or $\mathrm{n}(\%)$.

Table 2. Multivariable logistic regression associations of urinary phenols and parabens metabolites with CVD risk. 


\begin{tabular}{|c|c|c|c|c|c|}
\hline & Q1 & Q2 & Q3 & Q4 & $P$ value \\
\hline \multicolumn{6}{|l|}{ URBP3 } \\
\hline Model 1 & Ref & $0.83(0.70-0.99)$ & $0.84(0.69-1.01)$ & $0.74(0.61-0.91)$ & 0.019 \\
\hline Model 2 & Ref & $0.84(0.69-1.01)$ & $0.86(0.70-1.04)$ & $0.85(0.69-1.04)$ & 0.209 \\
\hline Model 3 & Ref & $0.84(0.70-1.02)$ & $0.88(0.72-1.08)$ & $0.88(0.71-1.09)$ & 0.325 \\
\hline \multicolumn{6}{|l|}{ URBPA } \\
\hline Model 1 & Ref & $1.13(0.94-1.35)$ & $0.99(0.81-1.21)$ & $1.39(1.15-1.68)$ & 0.001 \\
\hline Model 2 & Ref & $1.11(0.91-1.36)$ & $1.02(0.81-1.28)$ & $1.48(1.18-1.86)$ & 0.001 \\
\hline Model 3 & Ref & $1.13(0.93-1.38)$ & $1.04(0.82-1.30)$ & $1.52(1.20-1.91)$ & 0.001 \\
\hline \multicolumn{6}{|l|}{ URTCS } \\
\hline Model 1 & Ref & $0.83(0.70-0.99)$ & $0.84(0.69-1.01)$ & $0.74(0.61-0.91)$ & 0.019 \\
\hline Model 2 & Ref & $0.89(0.73-1.07)$ & $0.83(0.69-1.01)$ & $0.74(0.61-0.91)$ & 0.031 \\
\hline Model 3 & Ref & $0.90(0.74-1.08)$ & $0.83(0.68-1.02)$ & $0.74(0.59-0.92)$ & 0.053 \\
\hline \multicolumn{6}{|l|}{ URMPB } \\
\hline Model 1 & Ref & $0.92(0.77-1.11)$ & $0.81(0.67-0.99)$ & $0.82(0.67-1.00)$ & 0.117 \\
\hline Model 2 & Ref & $0.95(0.79-1.15)$ & $0.85(0.70-1.04)$ & $0.85(0.69-1.06)$ & 0.352 \\
\hline Model 3 & Ref & $0.95(0.77-1.17)$ & $0.87(0.67-1.12)$ & $0.91(0.68-1.23)$ & 0.754 \\
\hline \multicolumn{6}{|l|}{ URPPB } \\
\hline Model 1 & Ref & $0.98(0.82-1.18)$ & $0.90(0.74-1.09)$ & $0.81(0.66-0.99)$ & 0.167 \\
\hline Model 2 & Ref & $1.03(0.85-1.24)$ & $0.93(0.76-1.14)$ & $0.87(0.70-1.07)$ & 0.401 \\
\hline Model 3 & Ref & $1.07(0.87-1.32)$ & $1.02(0.80-1.32)$ & $0.96(0.72-1.29)$ & $0 . .832$ \\
\hline \multicolumn{6}{|c|}{ UR24-DCP } \\
\hline Model1 & Ref & $1.02(0.85-1.23)$ & $0.93(0.77-1.12)$ & $1.01(0.83-1.22)$ & 0.758 \\
\hline Model2 & Ref & $1.03(0.84-1.26)$ & $0.94(0.76-1.16)$ & $1.03(0.82-1.28)$ & 0.789 \\
\hline Model3 & Ref & $1.04(0.84-1.30)$ & $0.97(0.74-1.26)$ & $1.05(0.76-1.46)$ & 0.856 \\
\hline \multicolumn{6}{|c|}{ UR25-DCP } \\
\hline Model1 & Ref & $0.94(0.77-1.13)$ & $1.08(0.89-1.30)$ & $1.07(0.88-1.30)$ & 0.466 \\
\hline Model2 & Ref & $0.99(0.80-1.21)$ & $1.13(0.92-1.39)$ & $1.11(0.89-1.37)$ & 0.469 \\
\hline Model3 & Ref & $0.99(0.81-1.22)$ & $1.12(0.89-1.41)$ & $1.08(0.81-1.46)$ & 0.662 \\
\hline
\end{tabular}


Q, Quartitle; CVD, cardiovascular disease; URBP3, urinary benzophenone-3; URBPA, urinary bisphenol A; URTCS, urinary triclosan; URMPB, urinary methyl - paraben; URPPB, urinary propyl - paraben; UR24 - DCP, urinary 24 - dichlorophenol; UR25 - DCP, urinary 25 - dichlorophenol;

Model 1 was adjusted as age, sex, race, education levels and poverty;

Model 2 was adjusted as model 1 plus smoking, drinking, BMl, urinary creatinine, diabetes and hypertension;

Model3 was adjusted as model 2 plus other 6 heavy metals.

Table 3. WQS regression model to assess the adverse association of the mixture of urinary phenols and parabens with total CVD and individual CVDs risk.

\begin{tabular}{|llll|}
\hline Subgroup & OR & $95 \% \mathrm{Cl}$ & P value \\
\hline CVD & 1.16 & $(1.06-1.28)$ & 0.002 \\
\hline Congestive heart failure & 1.16 & $(0.96-1.41)$ & 0.132 \\
\hline Coronary heart disease & 1.18 & $(0.98-1.42)$ & 0.079 \\
\hline Angina & 1.30 & $(1.07-1.59)$ & 0.009 \\
\hline Heart attack & 1.30 & $(1.12-1.51)$ & $<0.001$ \\
\hline Stroke & 1.00 & $(0.78-1.29)$ & 0.971 \\
\hline
\end{tabular}

CVD, cardiovascular disease; WQS, weighted quantile sum; OR, odds ratio; $\mathrm{Cl}$, confidence interval;

WQS regression model was adjusted as age, sex, race, education levels, poverty, smoking, drinking, BMI, urinary creatinine, diabetes and hypertension.

\section{Figures}




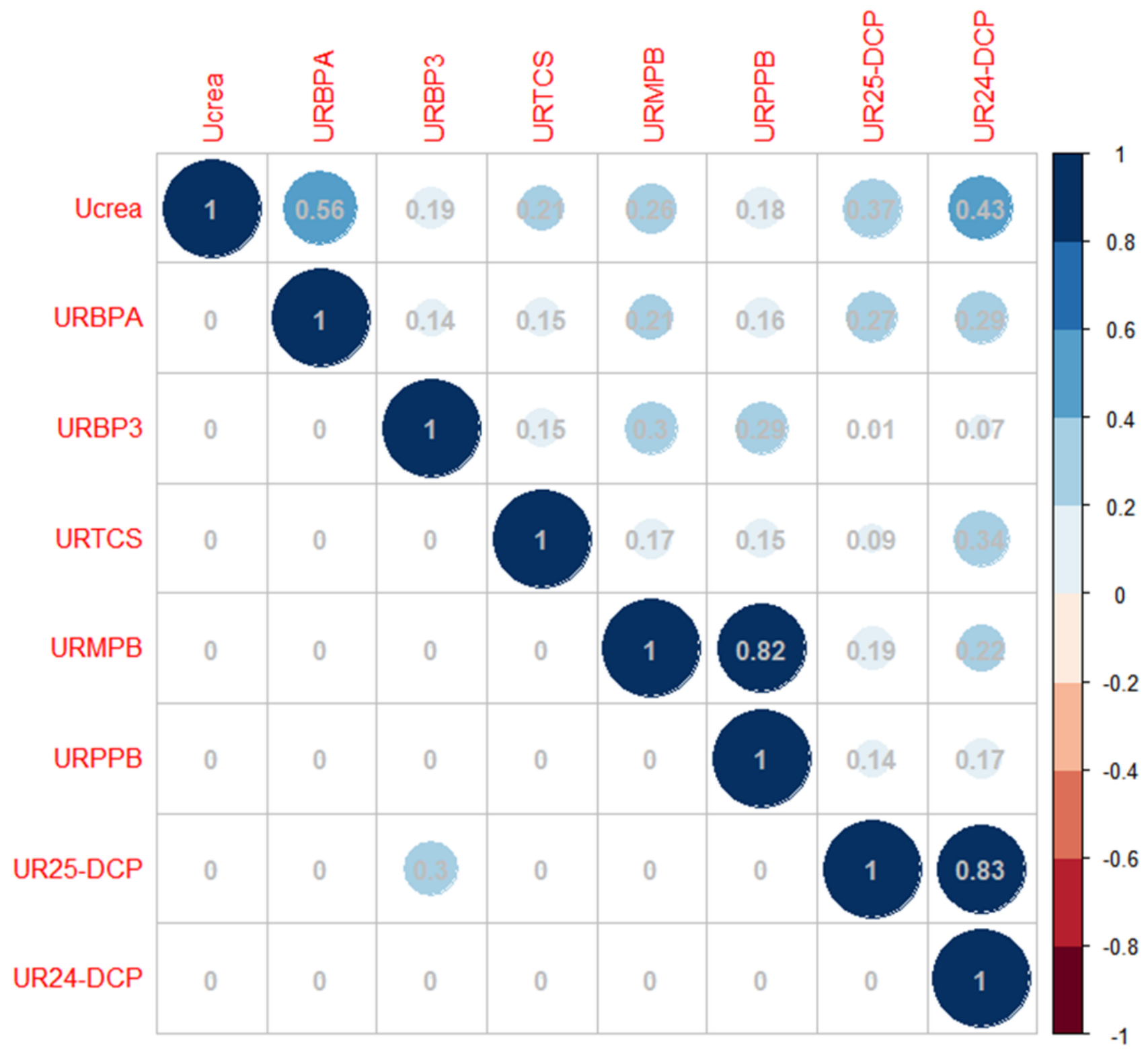

Figure 1

Pairwise Pearson correlation coefficients among urinary phenols and parabens metabolites concentrations in the United States general adult population (2005-2016). 


\section{Weights for Urinary Phenols and Parabens}

$\begin{array}{lll}\text { Cardiovascular.diseases } & \text { Coronary.heart.disease } & \text { Heart.attack } \\ \text { Congestive.heart.failure } & \text { Angina } & \text { Stroke }\end{array}$

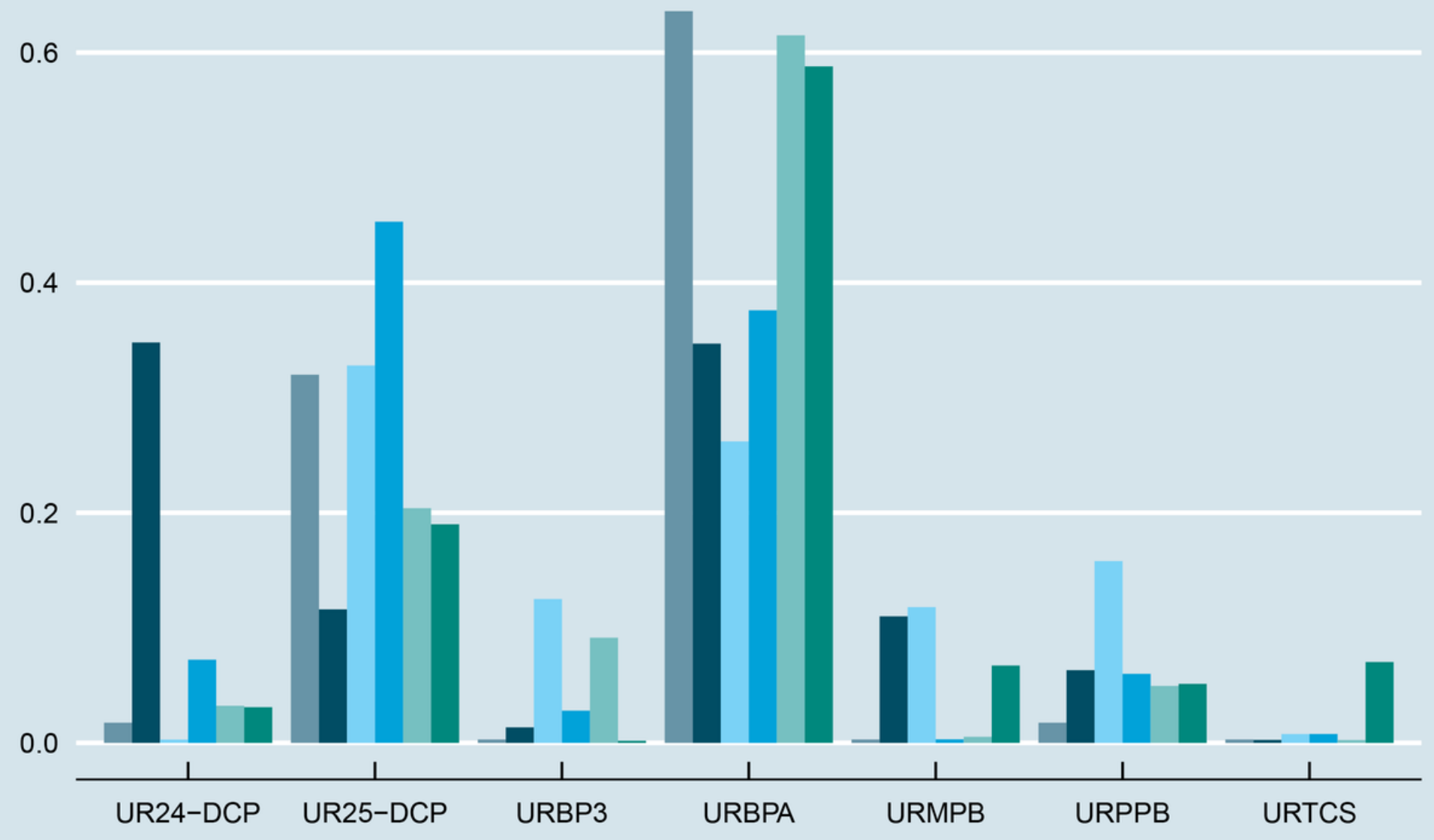

Figure 2

Weights from WQS regression for urinary phenols, parabens index and the prevalence of total and individual CVD. Protective models are shown and adjusted for age, sex, race, urinary creatinine, education levels, smoking, poverty, body mass index, diabetes mellitus and hypertension. WQS, weighted quantile sum; CVD, cardiovascular disease. 


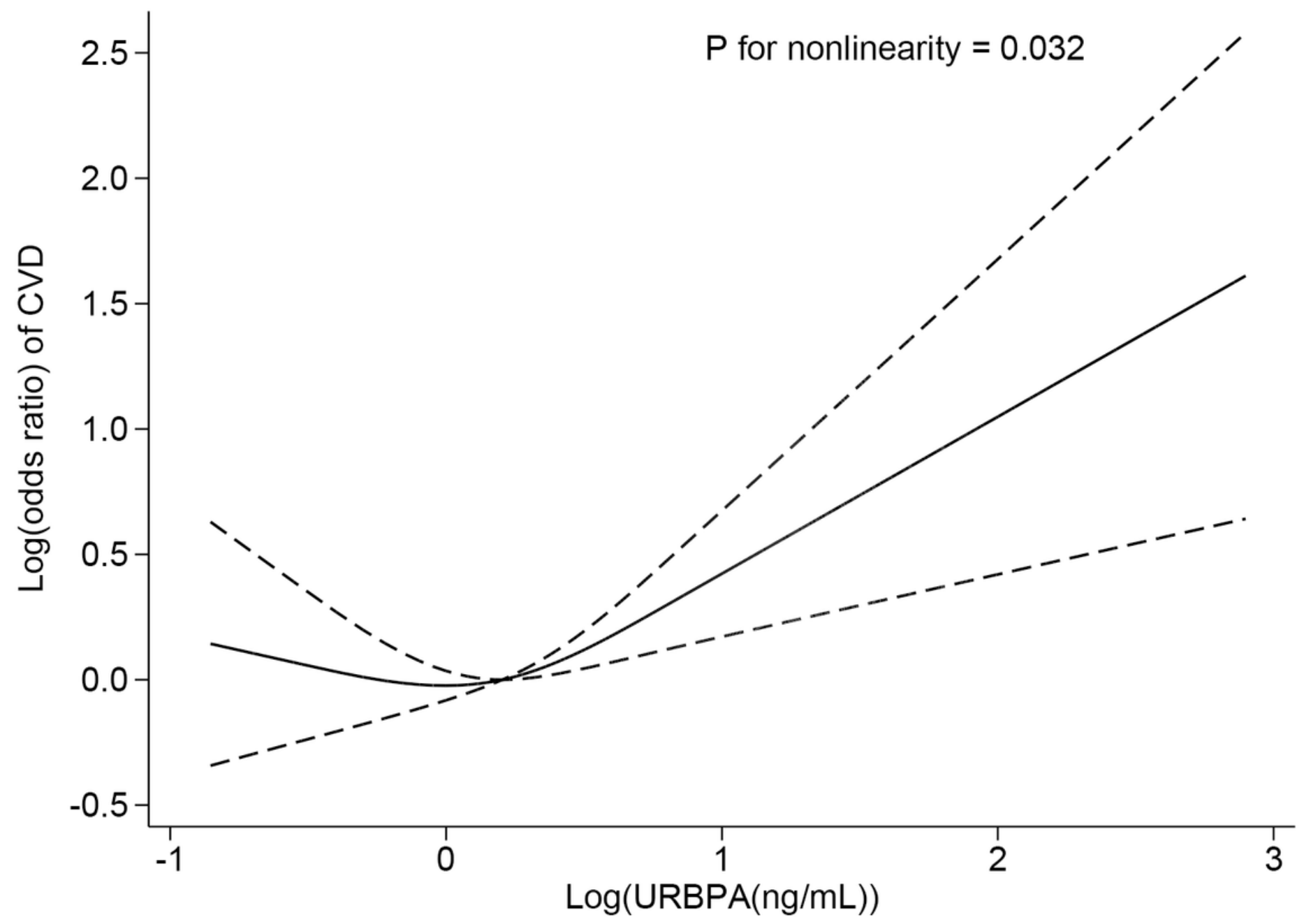

Figure 3

The restricted cubic spline with a multivariate logistic regression model shown association between URBPA and the prevalence rates of total CVD. URBPA, urinary bisphenol A; CVD, cardiovascular disease. 


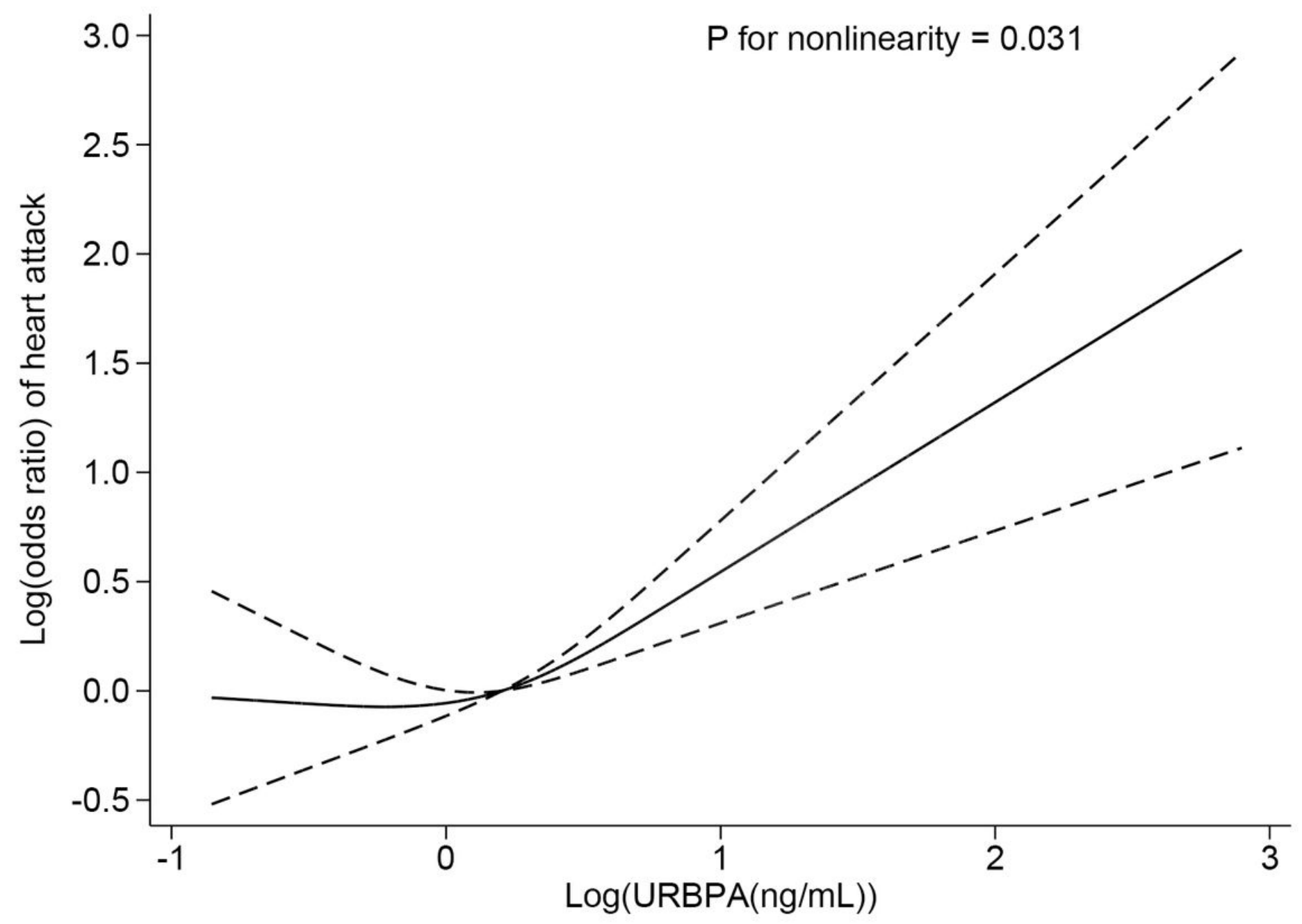

Figure 4

The restricted cubic spline with a multivariate logistic regression model shown association between URBPA and the prevalence rates of heart attack. URBPA, urinary bisphenol A.

\section{Supplementary Files}

This is a list of supplementary files associated with this preprint. Click to download.

- Fig.S1flowdiagram.jpg

- Fig.S2nonlinear.jpg

- SupplymentTables.docx 